(C) 2020 Universidad Nacional Autónoma de México, Facultad de Estudios Superiores Zaragoza.

Este es un artículo Open Access bajo la licencia CC BY-NC-ND (http://creativecommons.org/licenses/by-nc-nd/4.0/).

TIP Revista Especializada en Ciencias Químico-Biológicas, 23: 1-7, 2020.

https://doi.org/10.22201/fesz.23958723e.2020.0.246

\title{
Optimización del proceso de extracción asistida por ultrasonido de compuestos fenólicos de Justicia spicigera Schltdl. mediante la metodología de superficie de respuesta
}

\author{
Saúl Hernández-Rodríguez ${ }^{1}$, Cinthya Nathaly Quiroz-Reyes ${ }^{1}$, \\ María Eugenia Ramírez-Ortiz ${ }^{2}$, Elba Ronquillo-de Jesús ${ }^{3}$ \\ y Miguel Ángel Aguilar-Méndez ${ }^{1 *}$ \\ ${ }^{1}$ Instituto Politécnico Nacional, Centro de Investigación en Ciencia Aplicada y Tecnología \\ Avanzada, Laboratorio de Biomateriales. Legaria \# 694, Col. Irrigación, 11500, Ciudad de \\ México, México. ${ }^{2}$ Facultad de Estudios Superiores de Cuautitlán-UNAM, Campo 1, Edo. \\ de México, 54740, México. ${ }^{3}$ Universidad Politécnica de Francisco I. Madero, Dirección de \\ Ingeniería Agroindustrial, Tepatepec, 42660, Hidalgo, México. E-mail: *maguilarme@ipn.mx
}

\begin{abstract}
RESUMEN
El interés en el estudio de los polifenoles ha crecido de manera considerable en los últimos años debido a su alta capacidad para atrapar radicales libres asociados con diversas enfermedades. Por este motivo, el objetivo del presente trabajo fue obtener y caracterizar los compuestos fenólicos de Justicia spicigera Schltdl. mediante extracción asistida por ultrasonido. A los extractos, se les determinó el contenido fenólico total (método de Folin-Ciocalteu), actividad antioxidante (potencial antioxidante/reductor del hierro) y actividad secuestradora de radicales libres (método del radical libre 2,2-difenil-2-picrilhidrazil). La metodología de superficie de respuesta (MSR) se empleó para evaluar el efecto del disolvente (agua/etanol) y el tiempo de extracción, en el contenido de fenoles totales y las propiedades antioxidantes. El análisis de varianza (ANOVA) indicó que la concentración de agua en la mezcla del disolvente afectó de manera importante el rendimiento de los compuestos, así como la actividad antioxidante y antiradical. De acuerdo con la MSR, las condiciones óptimas para la extracción son $25 \%$ de agua en la mezcla del disolvente y un tiempo de sonicación de 16 minutos.
\end{abstract}

Palabras clave: muicle, polifenol, antioxidante, sonicación, diseño central compuesto.

Optimization of ultrasound-assisted extraction of phenolic compounds from Justicia spicigera Schltdl. by response surface methodology

\begin{abstract}
The interest in the study of polyphenols has grown considerably in recent years due to its high capacity to scavenge free radicals associated with various diseases. For this reason, the aim of this work was to obtain and characterize phenolic compounds from Justicia spicigera Schltdl by ultrasound-assisted extraction. The extracts were characterized in terms of the total phenolic content (Folin-Ciocalteu method), antioxidant activity (ferric-reducing/antioxidant power) and free radical scavenging activity (2,2-diphenyl-1-picrylhydrazyl free radical method). The response surface methodology (RSM) was used to evaluate the effect of the solvent (water/ethanol) and the extraction time, on the total phenolic content and antioxidant properties. The analysis of variance (ANOVA) indicated that the concentration of water in the solvent mixture significantly affected the yield of the compounds, as well as the antioxidant and antiradical activities. According to the RSM, the optimal extraction conditions are $25 \%$ water in the solvent mixture and a sonication time of 16 min. Keywords: muicle, polyphenol, antioxidant, sonication, central composite design.
\end{abstract}




\section{INTRODUCCIÓN}

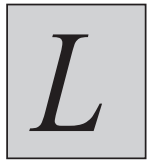

os polifenoles, son metabolitos secundarios producidos por las plantas, con al menos un anillo aromático y uno o más grupos hidroxilo. Algunos de ellos son moléculas simples de bajo peso molecular, mientras que otros forman polímeros (Stavrou, Christou \& Kapnissi-Christodoulou, 2018). Las frutas y vegetales son las principales fuentes de polifenoles. Los beneficios que el consumo de estos compuestos aportan a la salud están relacionados directamente con sus propiedades antioxidantes (Kabir et al., 2015). Diversos estudios han demostrado que poseen una gran capacidad para capturar radicales libres, que son los responsables del estrés oxidativo y ayudan en la prevención de diversas enfermedades crónico degenerativas como el cáncer, la ateroesclerosis, las enfermedades cardiovasculares y del sistema nervioso central (Khalifa, Zhu, Li, K \& Li, 2018; Majidinia, Bishayeeb \& Yousefic, 2019; Zhang, Qi \& Mine, 2019).

Justicia spicigera Schltdl. es una planta endémica de México, conocida comúnmente como muicle o muitle. Es un arbusto de hoja perenne con flores tubulares de color naranja (OrtizAndrade et al., 2012). En la medicina tradicional mexicana, $J$. spicigera ha sido una de las plantas más utilizadas debido a sus múltiples propiedades curativas. Investigaciones científicas han reportado la presencia de diversos compuestos fenólicos en extractos de diversas partes de la planta, siendo el flavonoide kaempferitrina uno de los más abundantes. A este compuesto se le ha asociado con propiedades antidiabéticas (Ortiz-Andrade et al., 2012), antitumorales y actividad inmunomoduladora (Alonso-Castro et al., 2012; Fernández-Pomares et al., 2018), actividad ansiolítica (García-Ríos, Mora-Pérez, GonzálezTorres, Carpio-Reyes \& Soria-Fregozo, 2019) y anticonvulsivo (González-Trujano et al., 2017).

A diferencia de los métodos de extracción convencionales, la extracción asistida por ultrasonido es un método simple y de bajo costo, que permite disminuir el tiempo y consumo del disolvente, con un aumento en el rendimiento de extracción (Živković, Janković, Menković \& Šavikin, 2019). La eficiencia de la extracción asistida por ultrasonido se debe a los efectos producidos por la cavitación acústica en el disolvente. Este efecto permite una mejor penetración del disolvente en la muestra y en consecuencia el mejoramiento de la transferencia de masa desde las membranas celulares (Da Porto, Porretto \& Decorti, 2013).

La extracción es la etapa inicial en la obtención de compuestos bioactivos. Diversos factores, como el tiempo de extracción, temperatura, tipo de disolvente y relación sólido/líquido pueden afectar el rendimiento y la bioactividad de los compuestos (Liu, Luo, Wang \& Yuan, 2019). En este sentido, es importante evaluar el efecto de estos factores mediante técnicas que permitan encontrar las condiciones ideales donde las pérdidas se minimicen. La metodología de superficie de respuesta(MSR) es una serie de técnicas matemáticas y estadísticas útiles en el modelado y el análisis de problemas en los que una respuesta de interés recibe la influencia de diversas variables y donde el objetivo es optimizar esta respuesta (Montgomery, 2005). Una de las principales ventajas de la MSR es el número reducido de experimentos necesarios para estudiar múltiples factores y sus interacciones (Cai, Chen, Yi \& Zou, 2019). Aunque existen algunos reportes sobre la extracción y evaluación de la actividad antioxidante de los compuestos fenólicos de la planta $J$ spicigera, no existe información relacionada con el efecto del disolvente y el tiempo de extracción en el rendimiento y actividad de los compuestos bioactivos.

En este sentido, el objetivo del presente trabajo fue elucidar mediante la MSR,el efecto de los factores antes mencionados en el contenido y actividad antioxidante de los compuestos fenólicos extraídos de las hojas de J. spicigera.

\section{MATERIALES Y MÉTODOS Reactivos y materiales}

El material vegetal se recolectó en la localidad de San Luis Huexotla, municipio de Texcoco de Mora, Estado de México $\left(19^{\circ} 29^{\prime} 23^{\prime \prime} \mathrm{N}, 9^{\circ} 53^{\prime} 37^{\prime \prime} \mathrm{O}\right)$. Los reactivos Folin-Ciocalteu, 2,4,6-Tris(2-piridil)-s-triazina (TPTZ), 2,2-difenil-1picrilhidrazilo(DPPH), cloruro férrico hexahidratado, catequina, ácido gálico y trolox se adquirieron de Sigma-Aldrich (EUA). Los ácidos clorhídrico y acético, el carbonato de sodio y acetato de sodio se compraron de JT Baker (México). Los disolventes utilizados fueron grado técnico.

\section{Preparación del material vegetal}

El material vegetal fue llevado al laboratorio de Biomateriales del CICATA IPN-Unidad Legaria, donde fue lavado con agua corriente, sanitizado en una solución de hipoclorito de sodio al $1 \%$ y secado bajo condiciones de sombra y temperatura ambiente. Una vez secas, las hojas fueron separadas de los tallos y pulverizadas en un molino de discos (Modelo 148-2, The Bauer Bros Co., EUA). El polvo obtenido fue tamizado (100 mesh) y almacenado en bolsas de plástico previo a su análisis.

\section{Extracción asistida por ultrasonido}

La extracción asistida por ultrasonido se realizó en un baño ultrasónico Elma-TI-H-5 (Alemania). El material en polvo se mezcló con el disolvente, en una relación 1:20 (sólido/líquido). El disolvente de extracción se conformó de una mezcla de agua/etanol a distintas proporciones (Tabla I). La mezcla resultante se acidificó con ácido fórmico al 1\% y se sometió a tratamiento ultrasónico a $25 \mathrm{kHz}$. Posteriormente, los extractos se centrifugaron ( 15 min a $1,750 \mathrm{rpm}$ ) y el sobrenadante se filtró y envasó. Los residuos se sometieron a una segunda extracción siguiendo las mismas condiciones experimentales. Finalmente, los sobrenadantes fueron mezclados y concentrados en un 
rotavapor (Yamato, RE500) para su posterior liofilización en un equipo Labconco (FreeZone 4.5 Liter).

Tabla I. Variables independientes y sus niveles de variación.

\begin{tabular}{|c|c|c|c|c|c|}
\hline $\begin{array}{c}\text { Variables } \\
\text { codificadas }\end{array}$ & $\mathbf{- 1 . 4 1}$ & $\mathbf{- 1}$ & $\mathbf{0}$ & $\mathbf{1}$ & $\mathbf{1 . 4 1}$ \\
\hline$X_{1}$ & 0 & 15 & 50 & 85 & 100 \\
\hline$X_{2}$ & 16 & 28 & 58 & 88 & 100 \\
\hline
\end{tabular}

$X_{1}$ : Concentración de agua (\%), $X_{2}$ : Tiempo de extracción (min).

\section{Análisis fitoquímico preliminar}

El análisis fitoquímico preliminar es una prueba cualitativa que indica la ausencia o presencia de los principales grupos de metabolitos presentes en un extracto vegetal. El análisis fitoquímico se realizó mediante métodos estándar (Takaidza, Mtunzi \& Pillay, 2018) y tomando en cuenta las siguientes pruebas cualitativas preliminares: reactivos de Dragendorff y Mayer para alcaloides, la prueba de Shinoda para flavonoides, las pruebas de Liebermann-Burchard y Salkowski para esteroles y terpenos, la prueba de $\mathrm{FeCl}_{3}$ para taninos y la prueba de la agitación para saponinas.

\section{Contenido fenólico total}

El contenido fenólico total se calculó a partir de la capacidad de reducción del reactivo de Folin-Ciocalteu (Waterhouse, 2002), con ligeras modificaciones. Un volumen de muestra de $20 \mu \mathrm{L}$ se adicionó a $1.4 \mathrm{~mL}$ de agua destilada, seguido de 100 $\mu \mathrm{L}$ del reactivo de Folin-Ciocalteu (2N). La solución se dejó reposar por 3 min a temperatura ambiente. Posteriormente, se adicionaron $300 \mu \mathrm{L}$ de una solución de carbonato de sodio al $20 \%(\mathrm{~m} / \mathrm{v})$ y $180 \mu \mathrm{L}$ de agua destilada. Después de $100 \mathrm{~min}$ de reposo en la oscuridad, la absorbancia se determinó a una longitud de onda de $760 \mathrm{~nm}$, utilizando un espectrofotómetro Multiskan Go (Thermo Fisher, Finlandia). Los resultados, para el ácido gálico, se expresaron en miligramos, equivalentes de ácido gálico, por gramo de materia seca (mg EAG / g MS).

\section{Capacidad antioxidante (FRAP)}

La capacidad antioxidante se determinó utilizando la prueba FRAP (ferric reduction antioxidant power). La determinación de FRAP está basada en la reducción del complejo $\mathrm{Fe}^{3+}-\mathrm{TPTZ}$ al complejo azulado $\mathrm{Fe}^{2+}$-TPTZ (Benzie \& Strain, 1996). La solución FRAP se preparó mezclando $25 \mathrm{~mL}$ de buffer de acetato $300 \mathrm{mM}$ (pH 3.6), $2.5 \mathrm{~mL}$ de solución TPTZ $(0.01 \mathrm{M})$ y 2.5 $\mathrm{mL}$ de una solución de $\mathrm{FeCl}_{3} \cdot 6 \mathrm{H}_{2} \mathrm{O}(0.02 \mathrm{M})$. La solución final se calentó a $37{ }^{\circ} \mathrm{C}$ por 4 min previo a su uso. Una alícuota de extracto de $100 \mu \mathrm{L}(1.0 \mathrm{mg} / \mathrm{mL})$ fue mezclada con $1,900 \mu \mathrm{L}$ de solución FRAP. Se dejó reposar por 30 min en la oscuridad y la absorbancia se determinó a $593 \mathrm{~nm}$. Los resultados se reportaron en micro moles equivalentes de trolox por cada 1,000 ppm ( $\mu \mathrm{M} \mathrm{ET} \mathrm{/} 1000$ ppm).

\section{Capacidad secuestradora de radicales libres (DPPH)}

La capacidad antiradical se cuantificó mediante el ensayo DPPH, siguiendo la metodología de Molyneux (2004), con algunas modificaciones. Una alícuota de $500 \mu \mathrm{L}$ de extracto se mezcló con $125 \mu \mathrm{L}$ Tris- $\mathrm{HCl}(0.1 \mathrm{M})$. A esta solución, se le agregaron $500 \mu \mathrm{L}$ de solución DPPH $(0.17 \mathrm{mg} / \mathrm{mL})$. Después de $30 \mathrm{~min}$ de reposo en la oscuridad, la absorbancia se registró a $517 \mathrm{~nm}$, empleando ácido ascórbico como control. El porcentaje de reducción del radical DPPH fue calculado usando la ecuación 1.

$$
\text { Inhibición }(\%)=\left[1-\frac{\text { Absorbancia de la muestra }}{\text { Absorbancia del control }}\right] * 100
$$

$\mathrm{El} \mathrm{EC}_{50}$ se define como la cantidad necesaria de extracto para reducir la concentración del radical DPPH en un $50 \%$. El valor de $\mathrm{EC}_{50}$ se calculó a partir de la gráfica del efecto de reducción del radical DPPH versus la concentración del extracto.

\section{Diseño de experimentos y análisis estadístico}

La extracción de los compuestos fenólicos de J. spicigera se realizó de acuerdo a lo establecido en un diseño experimental central compuesto, conformado de un factorial $2^{K}$ (donde $K=2$ ), una serie de corridas axiales y 5 puntos centrales. Las variables independientes o variables de estudio y sus niveles de variación se muestran en la Tabla I. El contenido de fenoles totales, la capacidad reductora del hierro (FRAP) y la actividad antiradical (DPPH) fueron seleccionadas como variables respuesta. Todos los experimentos se realizaron de manera aleatoria y los datos analizados mediante la MSR fueron para obtener los modelos matemáticos (ecuación 2) que describen las interacciones entre las variables independientes y las variables respuesta. $\mathrm{La}$ significancia de los modelos $(\mathrm{P}<0.05)$ se determinó mediante el ANOVA (prueba de $F$ ) y la influencia de los factores de estudio sobre las variables respuesta se observó a través de los gráficos de superficie de respuesta (Design Expert ver. 8, Stat-Ease Inc., EUA).

$$
Y=b_{0}+\sum_{i=1}^{2} b_{i} X_{i}+\sum_{i=1}^{2} b_{i i} X_{i}^{2}+\sum_{i=1}^{2} \sum_{j=i+1}^{2} b_{i j} X_{i} Y_{j}
$$

donde $Y$ es la variable respuesta, $b_{0}$ es el intercepto, $b_{i}, b_{i i}$ y $b_{i j}$ son los coeficientes de regresión y $X_{i}$ y $X_{j}$ son las variables de estudio.

Los análisis de regresión lineal se realizaron para evaluar la correlación entre el contenido de fenoles totales versus la actividad antioxidante (FRAP) y la actividad antiradical $\left(\mathrm{EC}_{50}\right)$.

\section{RESULTADOS Y DISCUSIÓN Perfil fitoquímico}

El perfil fitoquímico de los extractos crudos, acuosos y etanólicos de J. Spicigera (Tabla II) reveló la presencia importante de metabolitos secundarios como los flavonoides, 
esteroles y triterpenos. Por otra parte, los taninos y saponinas se identificaron en el extracto acuoso y etanólico, respectivamente. Estos resultados concuerdan con lo reportado previamente por García-Ríos et al. (2019).

Tabla II. Análisis fitoquímico preliminar de los extractos de $J$. spicigera.

\begin{tabular}{|c|c|c|}
\hline \multirow{2}{*}{ Tipo de metabolito } & \multicolumn{2}{|c|}{ Tipo de extracto } \\
\cline { 2 - 3 } & Acuoso & Etanólico \\
\hline Alcaloides & - & - \\
\hline Flavonoides & ++ & ++ \\
\hline Esteroles y terpenos & ++ & - \\
\hline Taninos & + & - \\
\hline Saponinas & - & + \\
\hline
\end{tabular}

+++ Abundante; ++ moderado; + presencia; - ausencia.

\section{Contenido fenólico total}

Con la finalidad de predecir la influencia de las variables de extracción en el contenido fenólico total, los resultados obtenidos (Tabla III) fueron analizados mediante la MSR. Una gráfica de superficie nos da una interpretación visual de las interacciones entre dos variables evaluadas y la relación entre la respuesta y los niveles experimentales de cada variable. De acuerdo con la Figura 1, el contenido fenólico total tendió a aumentar a medida que la concentración de agua en la mezcla del disolvente fue menor, independientemente del tiempo de extracción. Esto permite inferir que la mayoría de los compuestos fenólicos presentes en las hojas de muicle presentan una polaridad moderada al favorecerse su extracción

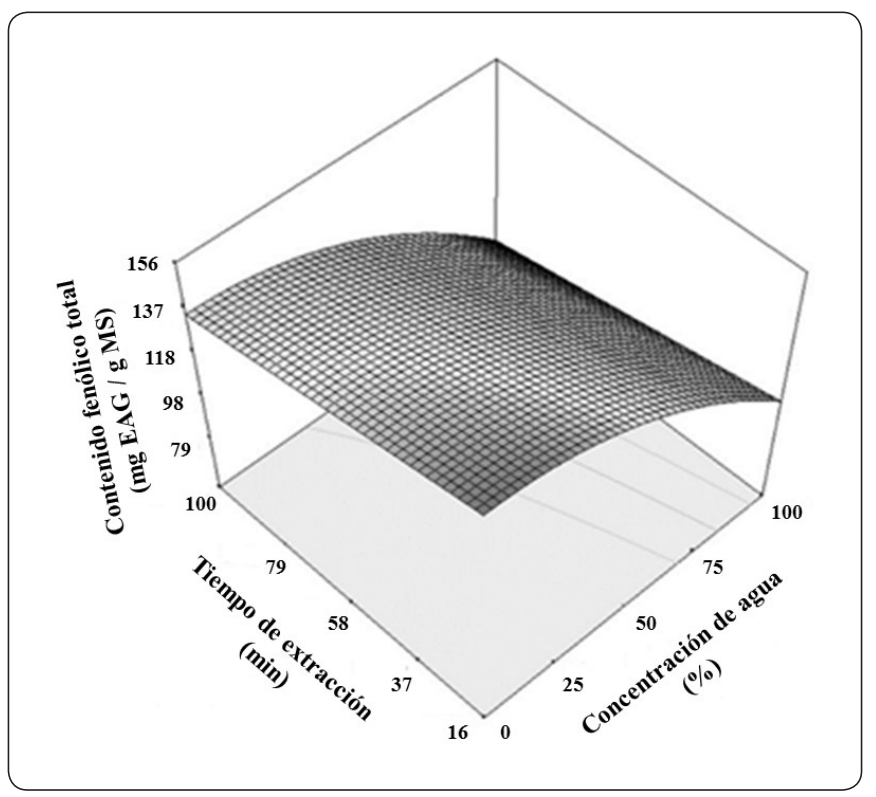

Figura 1. Superficie de respuesta mostrando el efecto de las variables de extracción en el contenido fenólico total.

cuando el etanol predomina en la mezcla de extracción. De acuerdo con Kelly, Kelly \& O’ Mahony (2019), los polifenoles presentan generalmente baja solubilidad en el agua, debido a su naturaleza química, mientras que son solubles preferentemente en disolventes de menor polaridad. Los resultados de esta investigación concuerdan con lo reportado por Mkaouar, Bahloul, Gelicus, Allaf \& Kechaou (2015), quienes señalan que los polifenoles de la hoja del olivo presentan una mayor afinidad con el etanol como disolvente de extracción.

Tabla III. Diseño experimental y resultados de las variables de estudio.

\begin{tabular}{|c|c|c|c|c|c|}
\hline Ensayo & $\begin{array}{c}\text { CA } \\
(\%)\end{array}$ & $\begin{array}{c}\text { Tiempo } \\
(\mathbf{m i n})\end{array}$ & $\begin{array}{c}\text { CFT } \\
(\mathbf{m g} \text { EAG / g MS) }\end{array}$ & $\begin{array}{c}\text { FRAP } \\
(\boldsymbol{\mu M} \text { ET / 1000 ppm) }\end{array}$ & $\begin{array}{c}\text { EC } \\
\mathbf{5 0} \\
(\mathbf{p p m})\end{array}$ \\
\hline 1 & 15 & 28 & 162.49 & 639.53 & 167.45 \\
\hline 2 & 85 & 28 & 109.67 & 546.19 & 423.46 \\
\hline 3 & 15 & 88 & 139.02 & 718.60 & 224.59 \\
\hline 4 & 85 & 88 & 84.89 & 472.98 & 585.65 \\
\hline 5 & 0 & 58 & 135.40 & 647.23 & 197.11 \\
\hline 6 & 100 & 58 & 100.93 & 424.90 & 860.46 \\
\hline 7 & 50 & 16 & 140.11 & 670.30 & 203.0 \\
\hline 8 & 50 & 100 & 133.22 & 605.18 & 266.92 \\
\hline 9 & 50 & 58 & 128.87 & 627.56 & 255.21 \\
\hline 10 & 50 & 58 & 125.62 & 679.22 & 279.89 \\
\hline 11 & 50 & 58 & 136.13 & 656.27 & 290.00 \\
\hline 12 & 50 & 58 & 127.44 & 595.01 & 282.90 \\
\hline 13 & 50 & 58 & 130.53 & 273.87 \\
\hline
\end{tabular}

CA: concentración de agua, CFT: contenido fenólico total, FRAP: actividad antioxidante, $\mathrm{EC}_{50}$ : actividad antiradical. 
La ecuación 3 representa el modelo matemático para la variable contenido de fenoles totales $\left(Y_{1}\right)$. Este modelo presentó un buen ajuste a los datos experimentales $\left(R^{2}=0.8359\right)$. Sin embargo, el ANOVA evidenció que solamente la variable $X_{1}$ (concentración de agua) fue estadísticamente significativa $(\mathrm{P}<0.001)$.

$$
Y_{1}=159.2872+6.9536 E^{-3} X_{1}-0.2440 X_{2}-5.5735 E^{-3} X_{1}^{2}
$$

\section{Capacidad reductora del hierro (FRAP)}

La capacidad reductora del hierro es considerada como la habilidad de un antioxidante natural para donar electrones (Wang, Huang, Shao, Qian \& Xu, 2012). La Figura 2 muestra el efecto de las condiciones de extracción en la capacidad reductora del hierro de los extractos de J. spicigera. Es posible observar que la capacidad reductora del hierro tiende a incrementarse cuando la concentración de agua en la mezcla del disolvente disminuye, observándose una ligera alza al aumentar el tiempo de extracción. Sin embargo, de acuerdo con el ANOVA, la variable tiempo no fue estadísticamente significativa $(\mathrm{P}>0.05)$. La Figura 3 muestra una correlación positiva, pero moderada entre el contenido de fenoles totales y la capacidad reductora del hierro $(r=0.81)$, lo que indica que aunque los compuestos fenólicos fueron en su mayoría los responsables de la actividad, también otros compuestos de naturaleza no fenólica, presentes en los extractos, contribuyeron con el poder antioxidante. Es muy probable que entre estos compuestos se encuentre el ácido ascórbico (vitamina C), ya que es un constituyente esencial que se encuentra prácticamente en todas las especies vegetales (Davey et al., 2000). De acuerdo con Paciolla et al. (2019), el ácido ascórbico puede actuar como un potente agente reductor, por

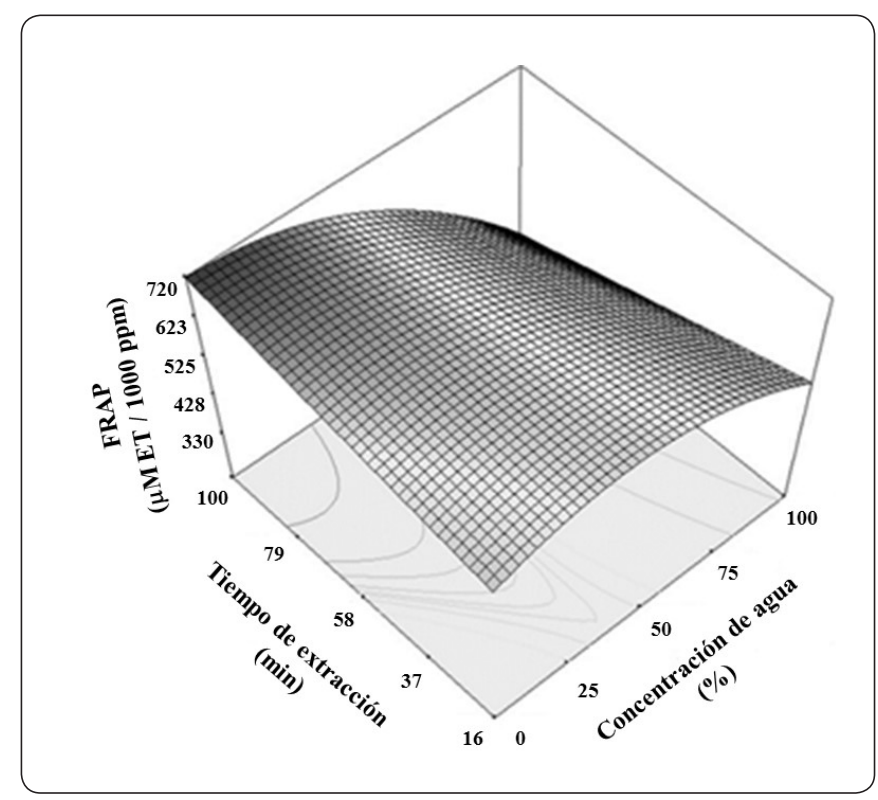

Figura 2. Superficie de respuesta mostrando el efecto de las variables de extracción en la actividad antioxidante (FRAP).

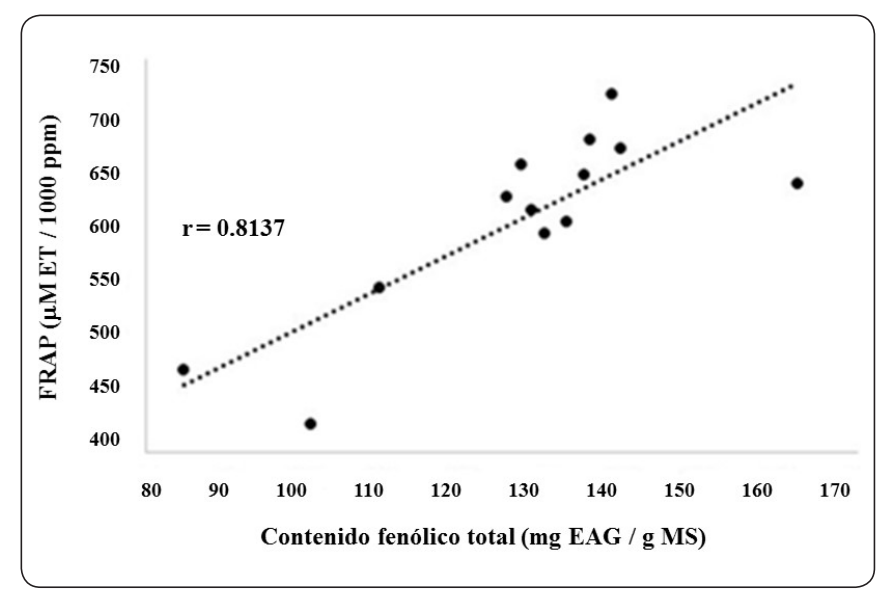

Figura 3. Correlación entre el contenido fenólico total y la capacidad antioxidante.

lo que tiene la capacidad de reducir el hierro, de la forma férrica $\left(\mathrm{Fe}^{3+}\right)$ a la ferrosa $\left(\mathrm{Fe}^{2+}\right)$.

El modelo que define a la variable FRAP mostró un buen ajuste a los datos experimentales $\left(R^{2}=0.9285\right)$. El término lineal y cuadrático de la variable concentración de agua, así como la interacción entre las dos variables fueron parámetros altamente significativos $(\mathrm{P}<0.01)$. La ecuación 4 muestra el polinomio de segundo orden con los términos significativos de la capacidad reductora del hierro $\left(Y_{2}\right)$ del extracto de $J$. spicigera.

$$
\begin{aligned}
Y_{2}= & 572.6519+3.6288 X_{1}-1.4496 X_{2} \\
& -0.0362 X_{1} X_{2}-0.0383 X_{1}^{2}
\end{aligned}
$$

\section{Capacidad antiradical (DPPH)}

La actividad secuestradora de los radicales libres, medida mediante el $\mathrm{EC}_{50}$ de los extractos de J. spicigera, fue determinada a través de la técnica de DPPH. La Figura 4 muestra la relación entre la capacidad antiradical y las condiciones de extracción. Se puede apreciar que el valor de $\mathrm{EC}_{50}$ fue menor al disminuir la proporción de agua en la mezcla del disolvente, independientemente del tiempo de extracción. El EC Es $_{50}$ está definido como la cantidad de extracto necesaria para disminuir la concentración inicial del radical DPPH en un $50 \%$. Entre menor sea el valor de $\mathrm{EC}_{50}$, mayor será la actividad de los extractos para actuar como secuestradores de radicales DPPH (Wootton-Beard, Moran \& Ryan, 2011).

La Figura 5 muestra una correlación negativa y moderada $(r=0.82)$ entre la actividad antiradical y el contenido fenólico total de los extractos de J. spicigera. Este resultado indica que la actividad antiradical no se limitó a la presencia de compuestos fenólicos, también otras moléculas con la propiedad de actuar como agentes antiradicales contribuyeron a esa actividad. De manera similar a la capacidad antioxidante, la vitamina $\mathrm{C}$ pudo contribuir en la actividad antiradical de los extractos de 
J. spicigera, ya que una de las funciones biológicas del ácido ascórbico en plantas y otros organismos es la de actuar como secuestrador de radicales libres (Davey et al., 2000).

El modelo matemático que expresa la variable respuesta $\mathrm{EC}_{50}$ $\left(Y_{3}\right)$, mostró un ajuste importante a los datos experimentales $\left(R^{2}=0.9415\right)$. Aunque el modelo fue estadísticamente significativo, el ANOVA indicó que sólo los términos lineal y cuadrático de la variable concentración de agua fueron significativos $(\mathrm{P}<0.05)$. La ecuación 5 muestra la ecuación polinomial cuadrática con los términos significativos que definen la capacidad antiradical de los extractos de J. spicigera.

$$
Y_{3}=149.9976+4.26280 X_{1}+1.3035 X_{2}+0.0976 X_{1}^{2}
$$

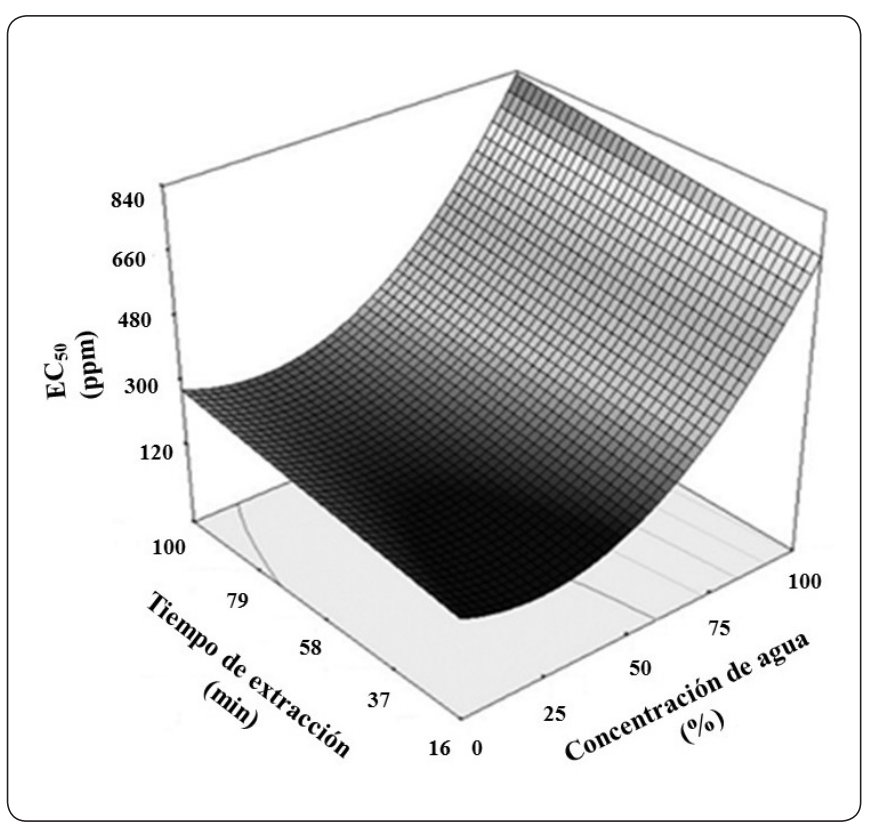

Figura 4. Superficie de respuesta mostrando el efecto de las variables de extracción en la capacidad inhibitoria de los radicales libres $\left(\mathrm{EC}_{50}\right)$.

\section{CONCLUSiOnes}

De $J$. spicigera fueron extraídos compuestos bioactivos con actividad antioxidante mediante extracción asistida por ultrasonido. El perfil fitoquímico evidenció principalmente la presencia de metabolitos secundarios como los flavonoides, esteroles y triterpenos. En el proceso de extracción, la relación agua/etanol fue el factor que afectó significativamente las variables de respuesta. La correlación entre el contenido de fenoles totales y la actividad antioxidante fue moderada, debida muy probablemente a la presencia de otros compuestos de naturaleza no fenólica que también contribuyeron con esta propiedad. De acuerdo con la MSR, las condiciones óptimas de extracción son una relación de 25/75 de agua/etanol como disolvente y un tiempo de sonicación de $16 \mathrm{~min}$.

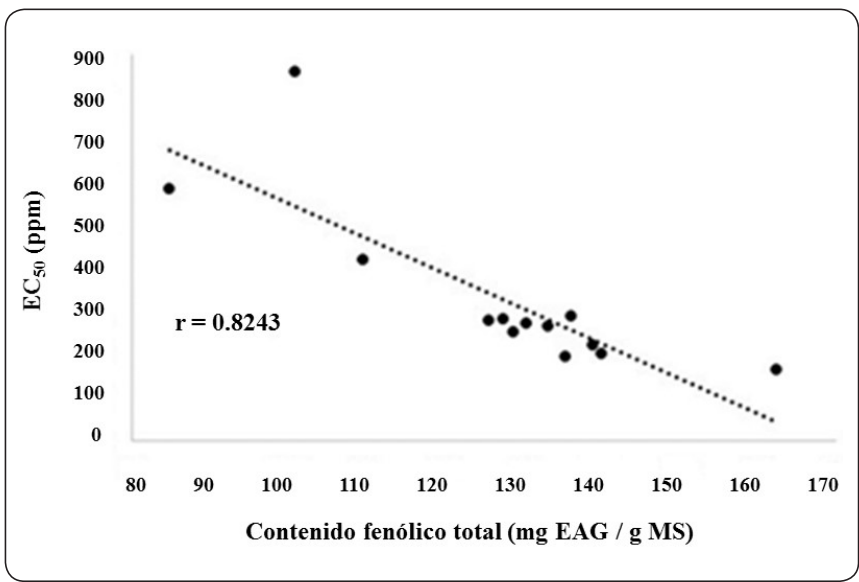

Figura 5. Correlación entre el contenido fenólico total y la actividad antiradical.

\section{Agradecimitentos}

Los autores agradecen al Instituto Politécnico Nacional por las facilidades y apoyos brindados para la realización de este trabajo. Saúl Hernández-Rodríguez agradece a CONACYT y a COFAA-IPN por las becas otorgadas.

\section{REFERENCIAS}

Alonso-Castro, A. J., Ortiz-Sánchez, E., Domínguez, F., Arana-Argáez, V., Juárez-Vázquez, M. C., Chávez, M., Carranza-Álvarez, C., Gaspar-Ramírez, O., EspinosaReyes, G., López-Toledo, G., Ortiz-Andrade, R. \& GarcíaCarrancá, A. (2012). Antitumor and immunomodulatory effects of Justicia spicigera Schltdl. (Acanthaceae). Journal of Ethnopharmacology, 141, 888-894. https://doi. org/10.1016/j.jep.2012.03.036.

Benzie, I. \& Strain, J. (1996). The ferric reducing ability of plasma (FRAP) as a measure of "antioxidant power": The FRAPAssay. Analytical Biochemistry, 239, 70-76. https:// doi.org/10.1006/abio.1996.0292.

Cai, L., Chen, B., Yi, F. \& Zou, S. (2019). Optimization of extraction of polysaccharide from dandelion root by response surface methodology: Structural characterization and antioxidant activity. International Journal of Biological Macromolecules, 140, 907-919. https://doi.org/10.1016/j. ijbiomac.2019.08.161.

Da Porto, C., Porretto, E. \& Decorti, D. (2013). Comparison of ultrasound-assisted extraction with conventional extraction methods of oil and polyphenols from grape (Vitis vinifera L.) seeds. Ultrasonics Sonochemistry, 20, 1076-1080. https://doi.org/10.1016/j.ultsonch.2012.12.002.

Davey, M. W., Montagu, M. V., Inzé, D., Sanmartin, M., Kanellis, A., Smirnoff, N., Benzie, I. J. J., Strain, J. J., Favell, D. \& Fletcher, J. (2000). Plant L-ascorbic acid: chemistry, function, metabolism, bioavailability and effects of processing. Journal of the Science of Food and Agriculture, 80, 825-860. https://doi.org/10.1002/(SICI)1097- 
0010(20000515)80:7<825::AID-JSFA598>3.0.CO;2-6.

Fernández-Pomares, C., Juárez-Aguilar, E., Domínguez-Ortiz, M. A., Gallegos-Estudillo, J., Herrera-Covarrubias, D., Sánchez-Medina, A., Aranda-Abreu, G. E., Manzo, J. \& Hernández, M. E. (2018). Hydroalcoholic extract of the widely used Mexican plant Justicia spicigera Schltdl. exerts a cytostatic effect on LNCaP prostate cancer cells. Journal of Herbal Medicine, 12, 66-72. https://doi.org/10.1016/j. hermed.2017.09.003.

García-Ríos, R. I., Mora-Pérez,A., González-Torres, D., CarpioReyes, R. J. \& Soria-Fregozo, C. (2019). Anxiolytic-like effect of the aqueous extract of Justicia spicigera leaves on female rats: A comparison to diazepam. Phytomedicine, 55, 9-13. DOI: 10.1016/j.phymed.2018.07.007.

González -Trujano, M. E., Domínguez, F., Pérez-Ortega, G., Aguillón, M., Martínez-Vargas, D., Almazán-Alvarado, S. \& Martínez, A. (2017). Justicia spicigera Schltdl. and kaempferitrin as potential anticonvulsant natural products. Biomedicine \& Pharmacotherapy, 92, 240-248. https:// doi.org/10.1016/j.biopha.2017.05.075.

Kabir, F., Tow, W. W., Hamauzu, Y., Katayama, S., Tanaka, S. \& Nakamura, S. (2015). Antioxidant and cytoprotective activities of extracts prepared from fruit and vegetable wastes and by-products. Food Chemistry, 167, 358-362. https://doi.org/10.1016/j.foodchem.2014.06.099.

Kelly, N. P., Kelly, A. L. \& O’Mahony, J. A. (2019). Strategies for enrichment and purification of polyphenols from fruitbased materials. Trends in Food Science \& Technology, 83, 248-258. https://doi.org/10.1016/j.tifs.2018.11.010.

Khalifa, I., Zhu, W., Li, K.-k. \& Li, C.-m. (2018). Polyphenols of mulberry fruits as multifaceted compounds: Compositions, metabolism, health benefits, and stability-A structural review. Journal of Functional Foods, 40, 28-43. https:// doi.org/10.1016/j.jff.2017.10.041.

Liu, X., Luo, G., Wang, L. \& Yuan, W. (2019). Optimization of antioxidant extraction from edible brown algae Ascophyllum nodosum using response surface methodology. Food and Bioproducts Processing, 114, 205-215. https://doi. org/10.1016/j.fbp.2019.01.003.

Majidinia, M., Bishayeeb,A.\& Yousefic, B. (2019). Polyphenols: Major regulators of key components of DNA damage response in cancer. DNA Repair, 82, 102679. https://doi. org/10.1016/j.dnarep.2019.102679.

Mkaouar, S., Bahloul, N., Gelicus, A., Allaf, K. \& Kechaou, N. (2015). Instant controlled pressure drop texturing far intensifying ethanol solvent extraction of olive (Olea europaea) leaf polyphenols. Separation and Purification Technology, 145, 139-146. https://doi.org/10.1016/j. seppur.2015.03.014.

Molyneux, P. (2004). The use of the stable free radical diphenylpicrylhydrazyl (DPPH) for estimating antioxidant activity. Songklanakarin Journal of Science Technology, 26, 211-219.

Montgomery, C. D. (2005). Diseño y análisis de experimentos. 2a ed. Limusa Wiley. Ciudad de México.

Ortiz-Andrade, R., Cabañas-Wuan, A., Arana-Argáez, V. E., Alonso-Castro, A. J., Zapata-Bustos, R., Salazar-Olivo, L. A., Domínguez, F., Chávez, M., Carranza-Álvarez, C. \& García-Carrancá, A. (2012). Antidiabetic effects of Justicia spicigera Schltdl. (Acanthaceae). Journal of Ethnopharmacology, 143, 455-462. https://doi. org/10.1016/j.jep.2012.06.043.

Paciolla, C., Fortunato, S., Dipierro, N., Paradiso, A., De Leonardis, D., Mastropasqua, L. \& de Pinto, M. C. (2019). Vitamin C in plants: from functions to biofortification. Antioxidants, 8,519.https://doi.org/10.3390/antiox8110519.

Stavrou, I. J., Christou, A. \& Kapnissi-Christodoulou, C. P. (2018). Polyphenols in carobs: A review on their composition, antioxidant capacity and cytotoxic effects, and health impact. Food Chemistry, 269, 355-374. https:// doi.org/10.1016/j.foodchem.2018.06.152.

Takaidza, S., Mtunzi, F. \& Pillay, M. (2018). Analysis of the phytochemical contents and antioxidant activities of crude extracts from Tulbaghia species. Journal of Traditional Chinese Medicine, 38, 272-279. https://doi.org/10.1016/j. jtcm.2018.04.005.

Wang, Y., Huang, S., Shao, S., Qian, L. \& Xu, P. (2012). Studies on bioactivities of tea (Camellia sinensis L.) fruit peel extracts: Antioxidant activity and inhibitory potential against $\alpha$-glucosidase and $\alpha$-amylase in vitro. Industrial Crops and Products, 37, 520-526. https://doi.org/10.1016/j. indcrop.2011.07.031.

Waterhouse, A. L. (2002). Determination of total phenolics. En: Current Protocols in Food Analytical Chemistry. R.E. Wrolstad (ed.), units I.1.1.1-I1.1.8. John Wiley and Sons. New York. https://doi.org/10.1002/0471142913.fai0101s06.

Wootton-Beard, P. C., Moran A. \& Ryan, L. (2011). Stability of the total antioxidant capacity and total polyphenol content of 23 commercially available vegetable juices before and after in vitro digestion measured by FRAP, DPPH,ABTS and Folin-Ciocalteu methods. Food Research International, 44, 217-224. https://doi.org/10.1016/j. foodres.2010.10.033.

Zhang, H., Qi, R. \& Mine, Y. (2019). The impact of oolong and black tea polyphenols on human health. Food Bioscience, 29, 55-61. https://doi.org/10.1016/j.fbio.2019.03.009.

Živković, J., Janković, T., Menković, N. \& Šavikin, K. (2019). Optimization of ultrasound-assisted extraction of isogentisin, gentiopicroside and total polyphenols from gentian root using response surface methodology. Industrial Crops \& Products, 139, 111567. https://doi.org/10.1016/j. indcrop.2019.111567. 